\title{
Age-related Differences in Countermovement Vertical Jump in Soccer Players 8-31 Years Old: the Role of Fat- free Mass
}

\author{
Nikolaidis P.T. ${ }^{1,2, *}$ \\ ${ }^{1}$ Department of Physical and Cultural Education, Hellenic Army Academy, Athens, Greece \\ ${ }^{2}$ Exercise Physiology Laboratory, Nikaia, Greece \\ *Corresponding author: pademil@hotmail.com
}

Received December 07, 2014; Revised February 18, 2014; Accepted February 24, 2014

\begin{abstract}
The aim of this study was to examine the effect of age on leg muscle power in male soccer players and to provide reference data. Male soccer players, all members of competitive soccer clubs and classified into six age groups (younger than $10 \mathrm{yr}, \mathrm{U10}, \mathrm{n}=17$; U12, $\mathrm{n}=27$; U14, $\mathrm{n}=70$; U16, $\mathrm{n}=$ 92; U18, $\mathrm{n}=33$; older than $18 \mathrm{yr}$, Adults, $\mathrm{n}=36$ ), participated in our study. They were examined for anthropometric characteristics, body composition, and performed the arm-swing countermovement vertical jump (CVJ) as a measure of leg muscle power. A one-way analysis of variance revealed significant differences between age groups with respect to CVJ $\left(\mathrm{F}_{5,269}=76.46, \mathrm{p}<\right.$ $0.001, \eta^{2}=0.59$ ). Our findings indicated that the older the age group, the higher the CVJ (e.g., $19.8 \pm 3.9 \mathrm{~cm}$ in U10, $30.0 \pm 5.9 \mathrm{~cm}$ in U14 and $41.8 \pm 6.0 \mathrm{~cm}$ in adult group). This result was in agreement with the significant and large correlation coefficient between age and CVJ $(r=0.68, p<0.001)$. Although the cross-sectional design of our study did not allow inferring causal relationship between age and CVJ, it is reasonable to support that there are large gains in leg muscle power with development in soccer players. These gains are accompanied by large increase of FFM during the same period. The findings of this study could be useful for coaches and trainers in the context of talent identification and training optimization.
\end{abstract}

Keywords: youth, lower limbs, muscle power, strength, football

Cite This Article: Nikolaidis P.T., "Age-related Differences in Countermovement Vertical Jump in Soccer Players 8-31 Years Old: the Role of Fat-free Mass.” American Journal of Sports Science and Medicine, vol. 2, no. 2 (2014): 60-64. doi: 10.12691/ajssm-2-2-1.

\section{Introduction}

In soccer, performance is influenced in great extent by leg muscle power [1]. In adolescence, this parameter of physical fitness is important in the context of talent identification and training monitoring. For instance, the measures of anaerobic power in young players aged 13-16 yr could partially determine if someone followed a career as an international, professional or amateur player in adulthood [2]. In spite of the popularity of soccer in adolescence, only a few studies have been carried out regarding physical traits and motor abilities of these athletes in a laboratory setting, especially with regard to leg muscle power.

We have recently investigated the development of anthropometric characteristics, body composition, physique [3], flexibility [4], isometric muscle strength [5], muscle endurance [6], force-velocity characteristics [7], performance in Wingate anaerobic test [8] and aerobic power in young soccer players [9]. The main findings of these studies revealed (a) significant differences between adolescent and adult players, (b) differences between the players in the lower spectrum and those in the higher spectrum of adolescence, and (c) better scores in soccer players than in general population.

Vertical jump has been used as a measure of leg muscle power in soccer $[10,11,12,13]$ and to identify bilateral differences [14,15]. Moreover, recent research has supported an association between vertical jump and sport performance; vertical jump correlated with both $10 \mathrm{~m}$ and $30 \mathrm{~m}$ sprint time $(\mathrm{r}=-0.72$ and $\mathrm{r}=-0.60, \mathrm{p}<0.001$, respectively) [13]. In the case of adolescent players, there is limited information on their leg muscle power assessed by a vertical jump test. For instance, only one research has been ever carried out in a large sample of young players, aged 11-17 yr [16]. However, that study was not able to identify significant differences among age groups partially due to the number of participants $(n=184)$, and did not examine its findings with regard to children and adult values.

New normative data would be helpful to coaches and fitness trainers in order to select players and evaluate their training. Therefore, the aim of this study was to examine two relevant research hypotheses with regard to vertical jump; (a) adolescent had higher scores than children and lower scores than adult players, (b) players in the lower spectrum were outscored by those in the higher spectrum of adolescence. 


\section{Methods}

Participants and procedures. In this investigation, a descriptive-correlation design was used to examine the effect of age on the arm-swing countermovement vertical jump (CVJ) across adolescence. Testing procedures were performed in the beginning of competition period of season 2011-12. Written informed consent was received from all players or parents after verbal explanation of the experimental design and potential risks of study. Although it is difficult to define adolescence in terms of chronological age, because of variation in time of its onset and termination, it has been suggested to range between 10 and 22 y in boys [17]. For the purpose of our study, we followed this definition. Male soccer players, all members of competitive soccer clubs and classified into six age groups (younger than $10 \mathrm{yr}, \mathrm{U} 10, \mathrm{n}=17$; U12, $\mathrm{n}=27$; $\mathrm{U} 14, \mathrm{n}=70$; U16, $\mathrm{n}=92$; U18, $\mathrm{n}=33$; older than $18 \mathrm{yr}$,
Adults, $\mathrm{n}=36$ ), participated in our study (Table 1). They visited our laboratory once; anthropometric and body composition data were obtained followed by the CVJ.

Protocols and equipment. Height and body mass were measured using a stadiometer (SECA, Leicester, UK) and an electronic scale (HD-351, Tanita, Illinois, USA), respectively. Percent of body fat was calculated from the sum of 10 skinfolds using a skinfold calliper (Harpenden, West Sussex, UK), based on the formula proposed by Parizkova [18]. The participants performed the countermovement jumps with arm-swing [19] twice with 1-min break between the two trials and the best score was retained for subsequent analysis. The height of each jump was estimated using the Opto-jump (Microgate Engineering, Bolzano, Italy) and was expressed as $\mathrm{cm}$. Measurements were recorded to the nearest $0.1 \mathrm{~cm}$. CVJ has been shown to be a reliable test $[20,21]$.

Table 1. Anthropometric data and vertical jump scores of participants.

\begin{tabular}{|c|c|c|c|c|c|c|c|}
\hline & \multicolumn{6}{|c|}{ Age groups } & \\
\hline & $\mathrm{U} 10(\mathrm{n}=17)$ & $\mathrm{U} 12(\mathrm{n}=27)$ & $\mathrm{U} 14(\mathrm{n}=70)$ & U16 (n=92) & U18 (n=33) & Adults $(n=36)$ & ANOVA \\
\hline Age (yr) & $9.1 \pm 0.6$ & $10.9 \pm 0.7$ & $13.1 \pm 0.5$ & $15.0 \pm 0.6$ & $17.0 \pm 0.5$ & $21.7 \pm 3.4$ & $\mathrm{~F}_{5,269}=342.30, \mathrm{p}<0.001 \eta^{2}=0.86$ \\
\hline Weight (kg) & $33.6 \pm 7.3$ & $42.5 \pm 9.0$ & $50.8 \pm 9.5$ & $62.6 \pm 8.4$ & $66.6 \pm 5.9$ & $74.1 \pm 8.4$ & $\mathrm{~F}_{5,269}=94.70, \mathrm{p}<0.001, \eta^{2}=0.64$ \\
\hline Height $(\mathrm{cm})$ & $137 \pm 6$ & $146 \pm 7$ & $160 \pm 9$ & $171 \pm 7$ & $175 \pm 6$ & $177 \pm 5$ & $\mathrm{~F}_{5,269}=139.14, \mathrm{p}<0.001, \eta^{2}=0.72$ \\
\hline $\operatorname{BMI}\left(\mathrm{kgm}^{-2}\right)$ & $17.8 \pm 2.4$ & $19.6 \pm 2.7$ & $19.7 \pm 2.6$ & $21.2 \pm 2.2$ & $21.9 \pm 1.8$ & $23.6 \pm 2.4$ & $\mathrm{~F}_{5,269}=21.82, \mathrm{p}<0.001, \eta^{2}=0.29$ \\
\hline $\mathrm{BF}(\%)$ & $16.4 \pm 4.4$ & $19.3 \pm 4.8$ & $16.1 \pm 5.1$ & $15.4 \pm 3.8$ & $14.4 \pm 3.3$ & $15.9 \pm 3.5$ & $\mathrm{~F}_{5,269}=83.40, \mathrm{p}<0.001, \eta^{2}=0.08$ \\
\hline $\mathrm{FM}(\mathrm{kg})$ & $5.7 \pm 2.8$ & $8.6 \pm 3.8$ & $8.5 \pm 4.0$ & $9.8 \pm 3.3$ & $9.7 \pm 2.8$ & $12.0 \pm 3.8$ & $\mathrm{~F}_{5,269}=9.15, \mathrm{p}<0.001, \eta^{2}=0.15$ \\
\hline FFM (kg) & $27.8(4.7)$ & $33.9(5.4)$ & $42.4(6.6)$ & $52.8(6.3)$ & $56.9(4.3)$ & $62.1(5.0)$ & $\mathrm{F}_{5,269}=154.08 \mathrm{p}<0.001, \eta^{2}=0.74$ \\
\hline CVJ (cm) & $19.8 \pm 3.9$ & $22.8 \pm 3.6$ & $30.0 \pm 5.9$ & $36.1 \pm 5.9$ & $40.8 \pm 5.6$ & $41.8 \pm 6.0$ & $\mathrm{~F}_{5,269}=76.46, \mathrm{p}<0.001, \eta^{2}=0.59$ \\
\hline
\end{tabular}

$\mathrm{BMI}=$ body mass index, BF = body fat, FM = fat mass, FFM = fat-free mass, CVJ = countermovement vertical jump.

Data and statistical analysis. Results were presented as of variance. Correction for multiple comparisons was mean \pm standard deviation. The effect of age on CVJ was examined by Pearson moment correlation coefficient $(r)$. Magnitude of correlation coefficients were considered as trivial $(\mathrm{r} \leq 0.1)$, small $(0.1 \leq \mathrm{r}<0.3)$ moderate $(0.3 \leq \mathrm{r}<$ $0.5)$, large $(0.5 \leq r<0.7)$, very large $(0.7 \leq r<0.9)$ and nearly perfect $(r \geq 0.9)$ and perfect $(r=1)$. Differences between age-groups were assessed using one-way analysis undertaken using the Bonferroni method. To interpret effect sizes for statistical differences in the ANOVA we used eta square classified as small $\left(0.010<\eta^{2} \leq 0.059\right)$, medium $\left(0.059<\eta^{2} \leq 0.138\right)$ and large $\left(\eta^{2}>0.138\right)$ [22]. Significance level was set at alpha $=0.05$. Statistical analyses were performed using IBM SPSS v.20.0 statistical software (SPSS Inc., Chicago, IL, USA).

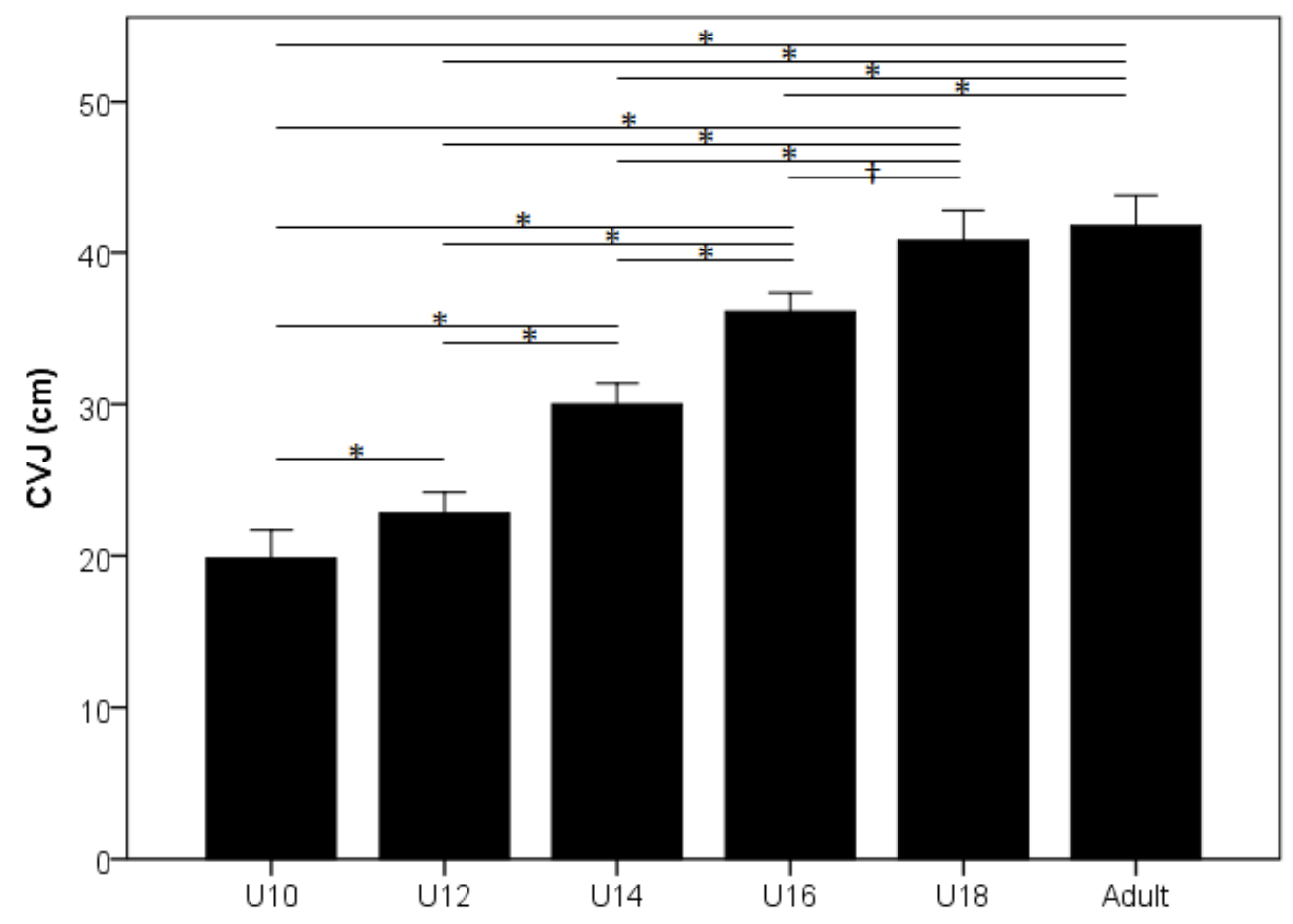

Figure 1. Differences in countermovement vertical jump (CVJ) between age groups. Error bars represent standard error. ${ }^{*} \mathrm{p}<0.001$, $\dagger \mathrm{p}<0.01$ 


\section{Results}

The basic characteristics of participants can be seen in Table 1. Briefly, our sample was consisted by U10 (6\%), U12 (10\%), U14 (26\%), U16 (34\%), U18 (12\%) and adult soccer players (13\%). There were significant differences between age groups for age, weight, height, BMI, BF and CVJ. The older the age group, higher values were recorded. The relationship between age groups and age with CVJ can be found in Figure 1 and Figure 2. Taking into account the eta square as a measure of effect size, there was a large effect of age groups on CVJ. Accordingly, there was a large correlation between age and CVJ ( $\mathrm{r}=0.68, \mathrm{p}<0.001)$. We found also a large correlation of $\mathrm{CVJ}$ with weight and fat-free mass (Figure 3).

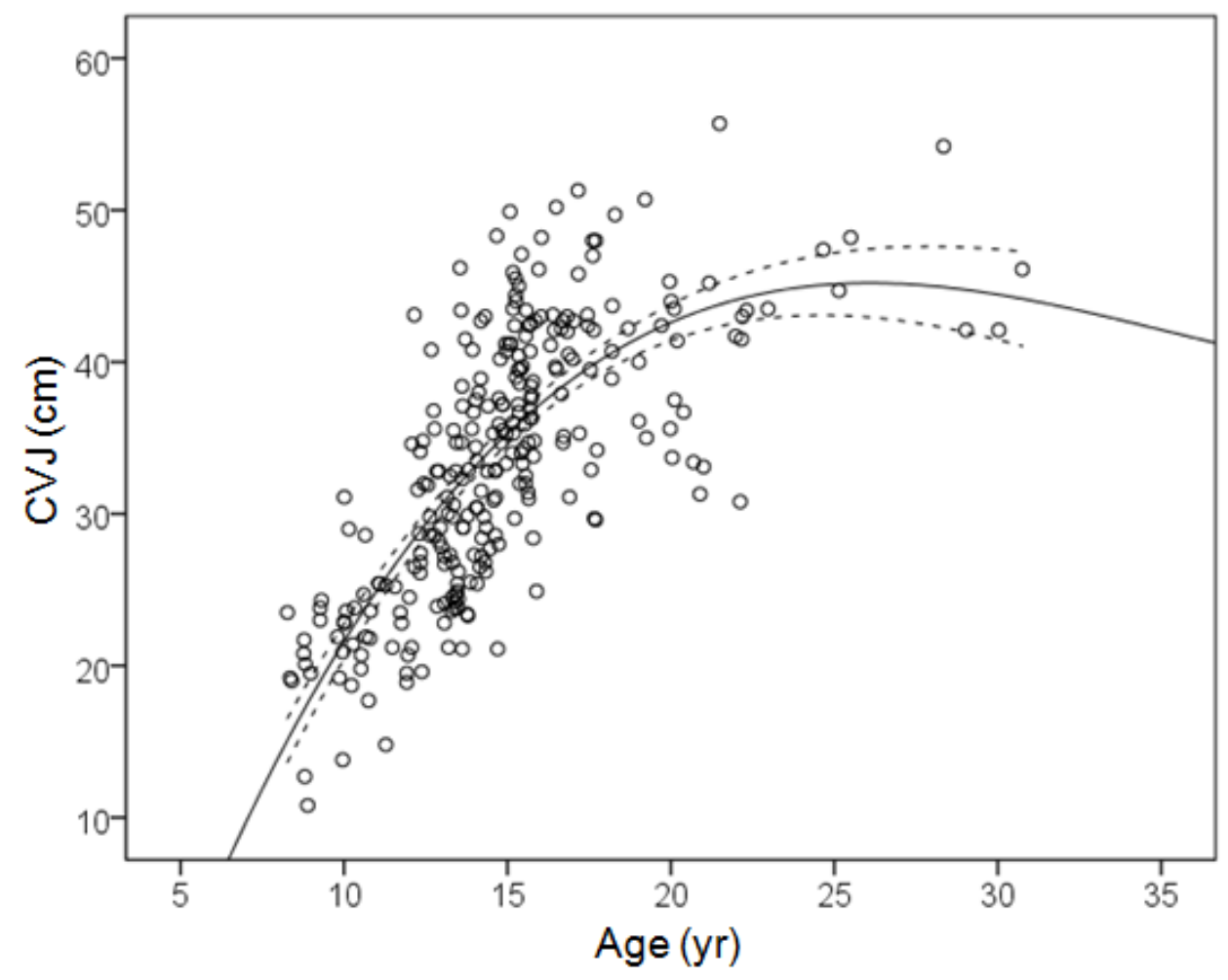

Figure 2. Relationship between age and countermovement vertical jump (CVJ) in participants. Dashed lines represent upper and lower 95\% confidence intervals of the mean

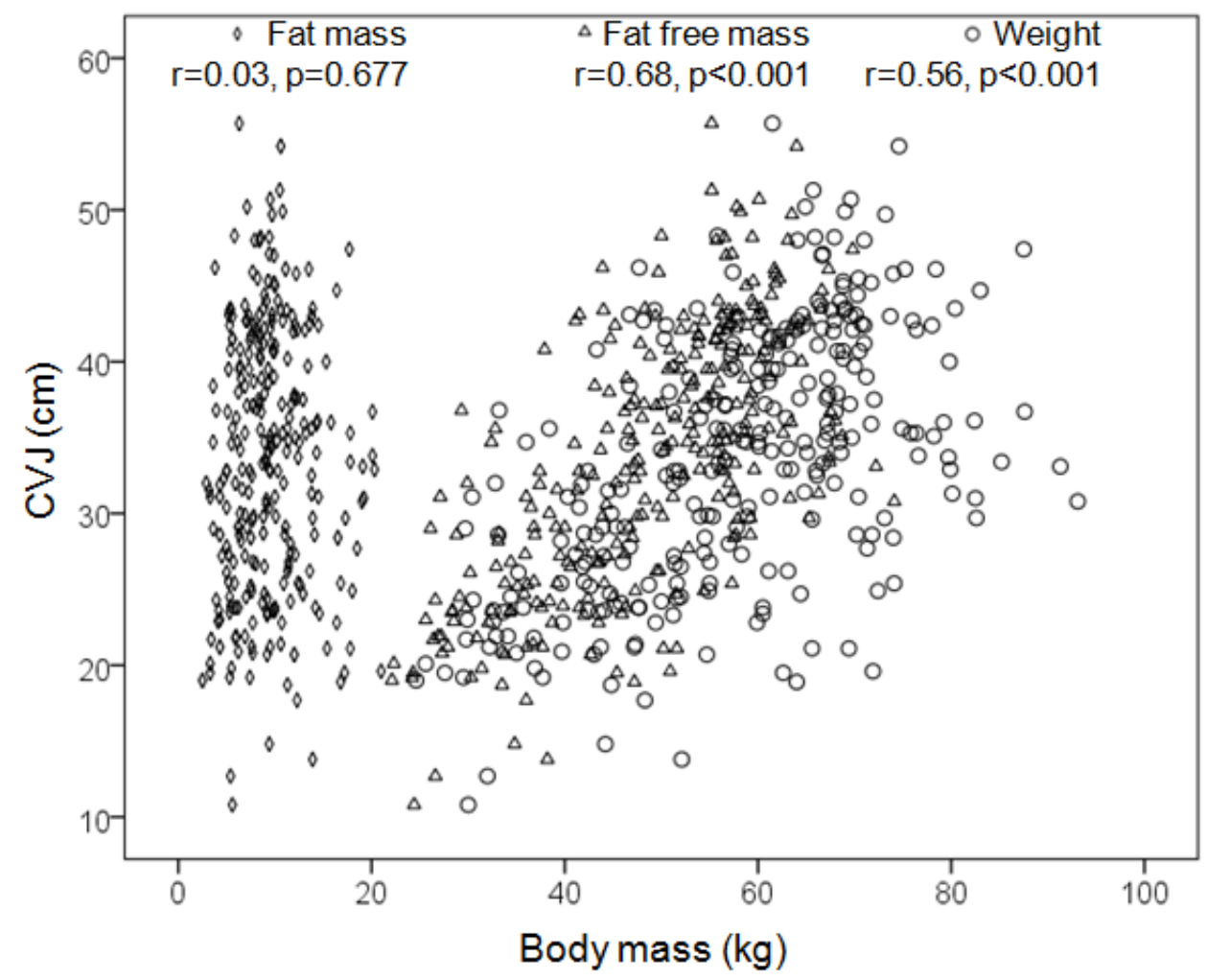

Figure 3. Relationship of countermovement vertical jump (CVJ) with fat mass, fat-free mass and total body weight 


\section{Discussion}

The main finding of this study was that age is a very important factor on leg muscle power and this relationship seems to be attenuated after the age of $18 \mathrm{yr}$. There was no difference in CVJ between U18 and adult players. The smallest statistical difference was observed between U10 and U12, while the largest difference was between U12 and U14, followed by that between U14 and U16.

Compared with previous data on French soccer players [16], participants had lower score; 22.8 vs. $31.0 \mathrm{~cm}$ in U12, 30.0 vs. $34.3 \mathrm{~cm}$ in U14, 36.1 vs. $41.2 \mathrm{~cm}$ in U16 and 40.8 vs. $45.5 \mathrm{~cm}$ in U18. While there was a consistent difference between these two studies, both revealed important increase across this range of age ( $79 \%$ vs. $\sim 47 \%$ ). The trend of CVJ increase during adolescence was similar with that reported in a recent study [23], which reported values of $24.2 \mathrm{~cm}, 27.7 \mathrm{~cm}, 32.6 \mathrm{~cm}, 36.7 \mathrm{~cm}$ and $36.7 \mathrm{~cm}$ in U11, U13, U15, U17 and U19 soccer players ( $\sim 52 \%)$, respectively, as well as consistently higher values in trained than in untrained controls.

The similar values in CVJ between U18 and adults were in agreement with previous research [23,24]. For instance, the members of Italian national teams U17, U20 and U21 had similar CVJ $(40.9 \mathrm{~cm}, 40.2 \mathrm{~cm}$ and $40.3 \mathrm{~cm}$, respectively) [24]. Our interpretation for this finding is that muscle leg power does not discriminate between matured players independently from whether they are adults or in the highest spectrum of adolescence. Previous research which compared elite and non-elite players by playing position found higher CVJ in elite players only in the case of goalkeepers [25] showing that this fitness parameter does not discriminate players by performance level. The exception in the case of goalkeepers might be attributed to that they usually have higher vertical jump values when compared to outfielders [26].

From a physiological point of view, the large gains in CVJ were explained from the increase in FFM across adolescence. This finding confirmed previous research that suggested a large correlation between FFM and CVJ in 7-13 yr boys [27] and the directly proportional changes in FFM and CVJ in 11-16 yr boys [28]. Therefore, an increase of leg muscle power should be expected during growth independently from the stimulus of soccer training.

\section{Conclusions}

Although the cross-sectional design of our study did not allow inferring causal relationship between age and CVJ, it is reasonable to support that there are large gains in leg muscle power with development in soccer players. These gains are accompanied by large increase of FFM during the same period. The findings of this study could be useful for coaches and trainers in the context of talent identification and training optimization.

\section{References}

[1] Bangsbo J, Mohr M, Krustrup P. Physical and metabolic demands of training and match-play in the elite football player. J Sports Sci. 2006; 24(7): 665-74.
[2] Le Gall F, Carling C, Williams M, Reilly T. Anthropometric and fitness characteristics of international, professional and amateur male graduate soccer players from an elite youth academy. J Sci Med Sport. 2010; 13: 90-5.

[3] Nikolaidis P, Karydis NV. Physique and body composition in soccer players across adolescence. Asian J Sports Med. 2011; 2(2): 75-82.

[4] Nikolaidis PT. Age-related differences of hamstring flexibility in male soccer players. Baltic Journal of Physical Activity. 2012; 4(2): $110-5$.

[5] Nikolaidis PT. Development of isometric muscular strength in adolescent soccer players. Facta Universitatis Series Physical Education and Sport. 2012; 10(3): 231-42.

[6] Nikolaidis P. Core stability of male and female football players. Biomed Hum Kinetics. 2010; 2: 30-3.

[7] Nikolaidis P. Age-related differences in force-velocity characteristics in youth soccer. Kinesiology. 2012; 44(2): 130-8.

[8] Nikolaidis P. Anaerobic power across adolescence in soccer players. Hum Movement. 2011; 12(4): 342-7.

[9] Nikolaidis P. Cardiorespiratory power across adolescence in male soccer players. Hum Physiol. 2011; 37(5): 636-41.

[10] Caldwell BP, Peters DM. Seasonal variation in physiological fitness of a semiprofessional soccer team. J Strength Cond Res. 2009; 23(5): 1370-7.

[11] Chlif M, Jullien H, Temfemo A, Mezouk A, Manouvrier C, Choquet D. Suivi physique et physiologique de footballeurs semiprofessionnels: vers un entraînement individualisé par poste. Sci Sports. 2010; 25: 132-8.

[12] Lago-Penas C, Casais L, Dellal A, Rey E, Dominguez E. Anthropometric and physiological characteristics of young soccer players according to their playing positions: relevance for competition success. J Strength Cond Res. 2011;25(12):3358-67

[13] Wisloff U, Castagna C, Helgerud J, Jones R, Hoff J. Strong correlation of maximal squat strength with sprint performance and vertical jump height in elite soccer players. Br J Sports Med. 2004; 38(3): 285-8.

[14] Menzel HJ, Chagas MH, Szmuchrowski LA, Araujo SRS, De Andrade AGP, De Jesus-Moraleida FR. Analysis of lower limb asymmetries by isokinetic and vertical jump tests in soccer players. Journal of Strength and Conditioning Research. 2013 //; 27(5): 1370-7.

[15] Sannicandro I, Rosa RA, De Pascalis S, Piccinno A. The determination of functional asymmetries in the lower limbs of young soccer players using the countermovement jump. The lower limbs asymmetry of young soccer players. Science and Sports. 2012 //; 27(6): 375-7.

[16] Hertogh C, Micallef JP, Mercier J. Puissance anaérobie maximale chez l'adolescent (étude transversale). Sci Sports. 1992; 7(4): 207 13.

[17] Malina RM, Bouchard C, Bar-Or O. Growth, maturation and physical activity. Champaign: Human Kinetics; 2004.

[18] Parizkova J. Lean body mass and depot fat during autogenesis in humans. In: Parizkova J, Rogozkin V, editors. Nutrition, Physical Fitness and Health: International Series on Sport Sciences. Baltimore: University Park Press; 1978.

[19] Aragon-Vargas LF. Evaluation of four vertical jump tests: Methodology, reliability, validity, and accuracy. Meas Phys Educ Exerc Sci. 2000; 4: 215-28.

[20] Richter A, Räpple S, Kurz G, Schwameder H. Countermovement jump in performance diagnostics: Use of the correct jumping technique. Eur J Sport Sci. 2012; 12(3): 231-7.

[21] Markovic G, Dizdar D, Jukic I, Cardinale M. Reliability and factorial validity of squat and countermovement jump tests. J Strength Cond Res. 2004 Aug; 18(3): 551-5.

[22] Cohen J. Statistical power analysis for the behavioral sciences. 2nd ed. Hillsdale, NJ: Lawrence Erlbaum Associates; 1988.

[23] Keiner M, Sander A, Wirth K, Schmidtbleicher D. Is there a difference between active and less active children and adolescents in jump performance? Journal of Strength and Conditioning Research. 2013 //; 27(6): 1591-6.

[24] Castagna C, Castellini E. Vertical jump performance in Italian male and female national team soccer players. Journal of Strength and Conditioning Research. 2013 //; 27(4): 1156-61.

[25] Rebelo A, Brito J, Maia J, Coelho-E-Silva MJ, Figueiredo AJ, Bangsbo J, et al. Anthropometric characteristics, physical fitness and technical performance of under-19 soccer players by competitive level and field position. International Journal of Sports Medicine. 2013 //; 34(4): 312-7. 
[26] Ziv G, Lidor R. Physical Characteristics, Physiological Attributes, and On-Field Performances of Soccer Goalkeepers. Int J Sports Physiol Perform. 2011; 6(4): 509-24.

[27] Aouichaoui C, Trabelsi Y, Bouhlel E, Tabka Z, Dogui M, Richalet JP, et al. The relative contributions of anthropometric variables to vertical jumping ability and leg power in Tunisian children. J Strength Cond Res. 2012 Mar; 26(3): 777-88.

[28] Temfemo A, Hugues J, Chardon K, Mandengue SH, Ahmaidi S. Relationship between vertical jumping performance and anthropometric characteristics during growth in boys and girls. European journal of pediatrics. 2009 Apr;168(4): 457-64. 